\title{
A test for the search for life on extrasolar planets
}

\section{Looking for the terrestrial vegetation signature in the Earthshine spectrum ${ }^{\star}$}

\author{
L. Arnold ${ }^{1}$, S. Gillet ${ }^{2}$, O. Lardière ${ }^{2}$, P. Riaud ${ }^{2,3}$, and J. Schneider ${ }^{3}$ \\ 1 Observatoire de Haute-Provence (OHP) CNRS, 04870 Saint-Michel-l'Observatoire, France \\ ${ }^{2}$ Laboratoire d'Interférométrie Stellaire et Exoplanétaire (LISE) CNRS, 04870 Saint-Michel-l'Observatoire, France \\ e-mail: sgohp@obs-hp.fr; lardiere@obs-hp.fr; riaud@obs-hp.fr \\ 3 Observatoire de Paris-Meudon, 92195 Meudon Cedex, France \\ e-mail: Jean.Schneider@obspm.fr; Pierre.Riaud@obspm.fr
}

Received 24 December 2001 / Accepted 6 May 2002

\begin{abstract}
We report spectroscopic observations (400-800 nm, $R \approx 100$ ) of Earthshine in June, July and October 2001 from which normalized Earth albedo spectra have been derived. The resulting spectra clearly show the blue colour of the Earth due to Rayleigh diffusion in its atmosphere. They also show the signatures of oxygen, ozone and water vapour. We tried to extract from these spectra the signature of Earth vegetation. A variable signal (4 to $10 \pm 3 \%$ ) around $700 \mathrm{~nm}$ has been measured in the Earth albedo. It is interpreted as being due to the vegetation red edge, expected to be between 2 to $10 \%$ of the Earth albedo at $700 \mathrm{~nm}$, depending on models. We discuss the primary goal of the present observations: their application to the detection of vegetation-like biosignatures on extrasolar planets.
\end{abstract}

Key words. astrobiology - stars: planetary systems

\section{Introduction}

The search for life on extrasolar planets has become a reasonable goal since the discovery of Earth-mass planets around a pulsar (Wolszczan \& Frail 1992) and Jupiter-mass planets around main-sequence stars (Udry \& Mayor 2001). Although the detection of Earth-mass planets is not foreseen before space missions (like COROT scheduled for 2004, Schneider et al. 1998), it is likely that a significant proportion of main sequence stars have Earth-like companions in their habitable zone. An important question is what type of biosignatures will unveil the possible presence of life on these planets.

Spectral signatures can be of two kinds. A first type consists of biological activity by-products, such as oxygen and its by-product ozone, in association with water vapour, methane and carbon dioxide (Lovelock 1975; Owen 1980; Angel et al. 1986). These biogenic molecules present attractive narrow molecular bands. This led in 1993 to the Darwin ESA project (Léger et al. 1996), followed by a similar NASA project, Terrestrial Planet Finder (TPF, Angel \& Woolf 1997; Beichman et al. 1999). But oxygen is

Send offprint requests to: L. Arnold,

e-mail: arnold@obs-hp.fr

* Based on observations collected at the Observatoire de HauteProvence (OHP-CNRS), France. not a universal by-product of biological activity as demonstrated by the existence of anoxygenic photosynthetic bacteria (Blankenship et al. 1995).

A second type of biosignature is provided by signs of stellar light transformation into biochemical energy, such as the planet surface colour from vegetation, whatever the bio-chemical details (Labeyrie 1999). This must translates into the planet reflection spectrum by some characteristic spectral features. This signature is in principle a more general biomarker than any biogenic gas such as oxygen, since it is a general feature of any photosynthetic activity (here leaving aside chemotrophic biological activity). Unfortunately, it is often not as sharp as single molecular bands: although it is rather sharp for terrestrial vegetation at $\approx 700 \mathrm{~nm}$ (Clark 1999; Coliolo et al. 2000, see Fig. 1), its wavelength structure can vary significantly among bacteria species and plants (Blankenship et al. 1995).

Before initiating a search for extrasolar vegetation, it is useful to test if terrestrial vegetation can be detected remotely. This seems possible as long as Earth is observed with a significant spatial resolution (Sagan et al. 1993), but is it still the case if Earth is observed as a single dot? A way to observe the integrated Earth is to observe the Earthshine with the Moon acting like a remote diffuse reflector illuminated by our planet. It has been proposed for some time (Arcichovsky 1912) to look for the vegetation colour in the Earthshine to use it as a reference 


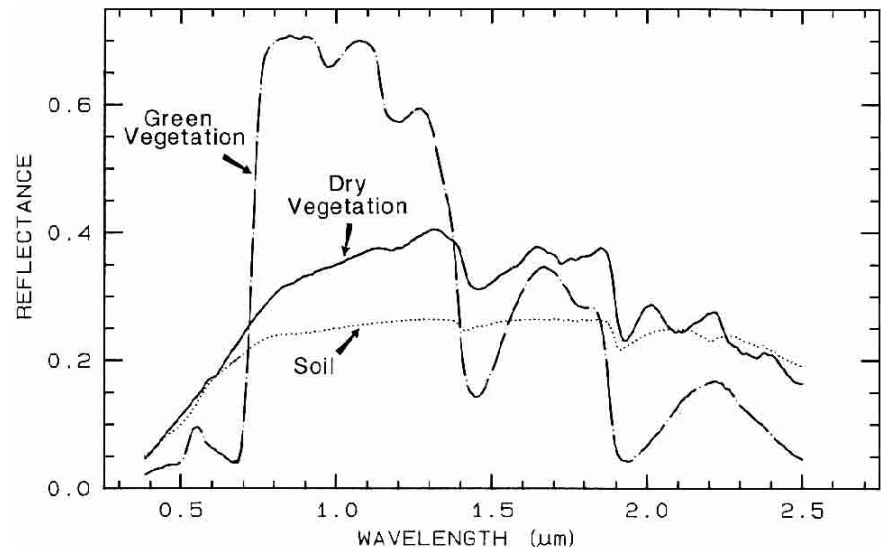

Fig. 1. Reflectance spectra of photosynthetic (green) vegetation, nonphotosynthetic (dry) and soil (from Clark 1999). The so-called vegetation red edge $(V R E)$ is the green vegetation reflectance strong variation from $\approx 5 \%$ at $670 \mathrm{~nm}$ to $\approx 70 \%$ at $800 \mathrm{~nm}$.

for the search of chlorophyll on other planets, but up to now, Earthshine observations apparently did not have sufficient spectral resolution for that purpose (Tikhoff 1914; Danjon 1928; Goode et al. 2001). We present in Sect. 3.1 normalized Earth albedo spectra showing several atmospheric signatures. We show in Sect. 3.2 how the vegetation signature around $700 \mathrm{~nm}$ can be extracted from these spectra.

\section{Observations and data reduction}

After a first test made in 1999 with the FEROS spectrograph $(R=48000)$ on the La Silla ESO $1.5 \mathrm{~m}$ telescope, we have built a dedicated spectrograph mounted on the $80 \mathrm{~cm}$ telescope at the Observatoire de Haute-Provence (Table 1).

We have observed the Moon at ascending and descending phases from April to October 2001. Data collected in June, July and October (Table 2) have been of sufficient quality to derive the results described in this article.

Our observation procedure is the following. The long spectrograph slit allows us to record simultaneously the Earthshine and sky background spectra ( $\approx 80 \mathrm{CCD}$ lines for each). This single exposure is bracketed by two spectra of the Moonlight. Each of the latter is the mean of 10 spectra (totalling $20^{\prime}$ ) taken in different regions to smooth the Moon albedo spatial variations. A series of flat fields (tungsten lamp) is recorded just after the previous cycle.

Before getting a final spectrum from binning of CCD lines, a sub-pixel alignment of CCD lines is done to correct the residual angle between the pixel rows and the dispersion direction: Each image is oversampled by a factor of 8 in the dispersion direction. Each line $i$ is then translated to maximize the crosscorrelation function from line $i$ with line 1 . After the line binning is done, the spectrum is resampled with $1 \mathrm{~nm} / \mathrm{pixel}$ for convenience.
Table 1. Telescope and spectrograph characteristics.

\begin{tabular}{ll}
\hline \hline Parameters & Values \\
\hline Telescope diameter, $f /$ ratio & $80 \mathrm{~cm}, 16.5$ \\
Slit length (unvignetted) & 6 arcmin $(2 \operatorname{arcmin})$ \\
Slit width & 1.6 to 7.8 arcsec \\
Spatial sampling & 1.4 arcsec $/$ pixel \\
Transmission grating & 100 lines $/ \mathrm{mm}$ \\
Spectral resolution $\lambda / \Delta \lambda$ & $\approx 50$ to $240 \mathrm{at} \lambda=700 \mathrm{~nm}$ \\
Max. spectral range & 400 to $900 \mathrm{~nm}$ \\
Spectral sampling & $2.6 \mathrm{~nm} / \mathrm{pixel}$ \\
CCD Kodak ship & non-ABG KAF-0401E \\
\hline
\end{tabular}

Table 2. Earthshine observations journal.

\begin{tabular}{llccl}
\hline \hline $\begin{array}{l}\text { Date } \\
\text { (yyyy/mm/dd) }\end{array}$ & $\begin{array}{l}\text { Hour } \\
\text { (h min UT) }\end{array}$ & $\begin{array}{l}\text { Exposure } \\
\text { time (s) }\end{array}$ & $\begin{array}{l}\text { Resolution } \\
\lambda / \Delta \lambda\end{array}$ & $\begin{array}{l}\text { Signal } \\
\text { to Noise }\end{array}$ \\
\hline $2001 / 06 / 17$ & 0237 to 0308 & 480 & 120 & 190 \\
$2001 / 06 / 18$ & 0249 to 0307 & 360 & 120 & 130 \\
$2001 / 06 / 24$ & 2020 to 2103 & 600 & 120 & 210 \\
$2001 / 06 / 26$ & 2041 to 2145 & 900 & 120 & 150 \\
$2001 / 07 / 23$ & 2004 to 2037 & 240 & 50 & 170 \\
$2001 / 07 / 24$ & 1954 to 2056 & 1080 & 50 & 390 \\
$2001 / 07 / 25$ & 1957 to 2124 & 1440 & 50 & 250 \\
$2001 / 10 / 13$ & 0216 to 0500 & 2640 & 240 & 240 \\
$2001 / 10 / 14$ & 0335 to 0404 & 480 & 240 & 100 \\
$2001 / 10 / 19$ & 1737 to 1754 & 240 & 240 & 40 \\
$2001 / 10 / 21$ & 1739 to 1925 & 1680 & 240 & 150 \\
\hline
\end{tabular}

${ }^{a}$ Cumulative exposure time from several $120 \mathrm{~s}, 180 \mathrm{~s}$ or $240 \mathrm{~s}$ single exposures.

${ }^{b}$ Signal to noise ratio at $\lambda=650 \mathrm{~nm}$ for the cumulative spectrum obtained after single images addition and lines binning.

\section{Results}

\subsection{Earth albedo $E A(\lambda)$}

Let us define the following spectra: we call the Sun as seen from outside the Earth atmosphere $S(\lambda)$, Earth atmosphere transmittance $A T(\lambda)$, Moonlight $M S(\lambda)$, Earthshine $E S(\lambda)$, Moon albedo $M A(\lambda)$, and Earth albedo $E A(\lambda)$. We have

$M S(\lambda)=S(\lambda) \times M A(\lambda) \times A T(\lambda) \times g_{1}$,

$E S(\lambda)=S(\lambda) \times E A(\lambda) \times M A(\lambda) \times A T(\lambda) \times g_{2}$.

The Earth albedo is simply given by Eq. (2)/Eq. (1), i.e.

$E A(\lambda)=\frac{E S(\lambda) \times g_{1}}{M S(\lambda) \times g_{2}}$

Simplifying by $A T(\lambda)$ means that $E S(\lambda)$ and $M S(\lambda)$ should be ideally recorded simultaneously to avoid significant airmass variation and thus Rayleigh scattering bias. The mean of the two $M S$ spectra bracketing $E S(\lambda)$ is thus used to compute $E A(\lambda)$. The $g_{i}$ terms are geometric factors related to the Sun, Earth and Moon positions. For simplicity, we set $g_{1}$ and $g_{2}$ equal to 1 , equivalent to a spectrum normalization. 


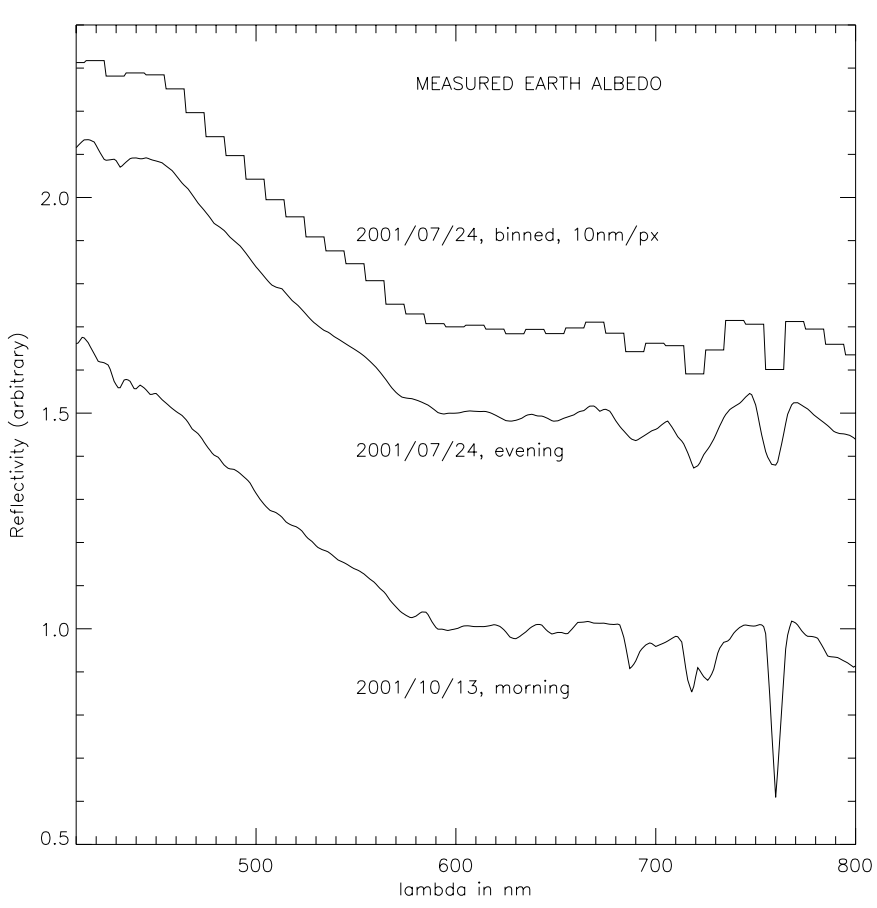

Fig. 2. Examples of measured Earth albedo spectra. Both spectra are normalized to 1 at $600 \mathrm{~nm}$, but the July spectrum is shifted upwards by 0.5 for clarity. The spectral resolution was $\approx 50$ in July, and $\approx 240$ in October. The July spectrum has been binned to $10 \mathrm{~nm} / \mathrm{px}$ to mimic the low resolution that might be used for the first extrasolar planet spectrum.

Figure 2 shows that the Earth should be seen as a blue object from space. This blue colour has been known for a long time, and has been confirmed by the Apollo astronauts (Kelley 1988). Tikhoff (1914) had already discovered the blue colour of Earthshine and interpreted it as being due to the Rayleigh scattering in the atmosphere. This point will be discussed in more details in Sect. 3.2.

The $\mathrm{H}_{2} \mathrm{O}$ bands around 690 and $720 \mathrm{~nm}$, and $\mathrm{O}_{2}$ narrower band at $760 \mathrm{~nm}$ are clearly visible with a resolution of $R \approx$ 50 . The slope variation occurring at $\approx 600 \mathrm{~nm}$ is partially the signature of the deepest zone of the broad ozone absorption band (Chappuis band), going from 440 to $760 \mathrm{~nm}$.

\subsection{Earth surface reflectance $S R(\lambda)$}

To detect the vegetation signature at $700 \mathrm{~nm}$, it is necessary to extract the Earth surface reflectance $S R(\lambda)$, that contains the spectral information on vegetation, from the atmosphere features contained in $A T(\lambda)$. Said differently, it is necessary to remove the atmospheric bands in this spectral region. Surface reflectance $S R(\lambda)$ is usually presented in Earth remote sensing science by a vector giving the directional properties of the scattered light (Liang \& Strahler 1999, BRDF 2000). But here, we adopt a simpler scalar definition allowing us to write the albedo spectrum $E A(\lambda)$ as the product

$E A(\lambda) \approx S R(\lambda) \times A T^{\alpha=2}(\lambda)$

meaning that photons are transmitted once through a one airmass Earth atmosphere, are scattered by the Earth's surface,

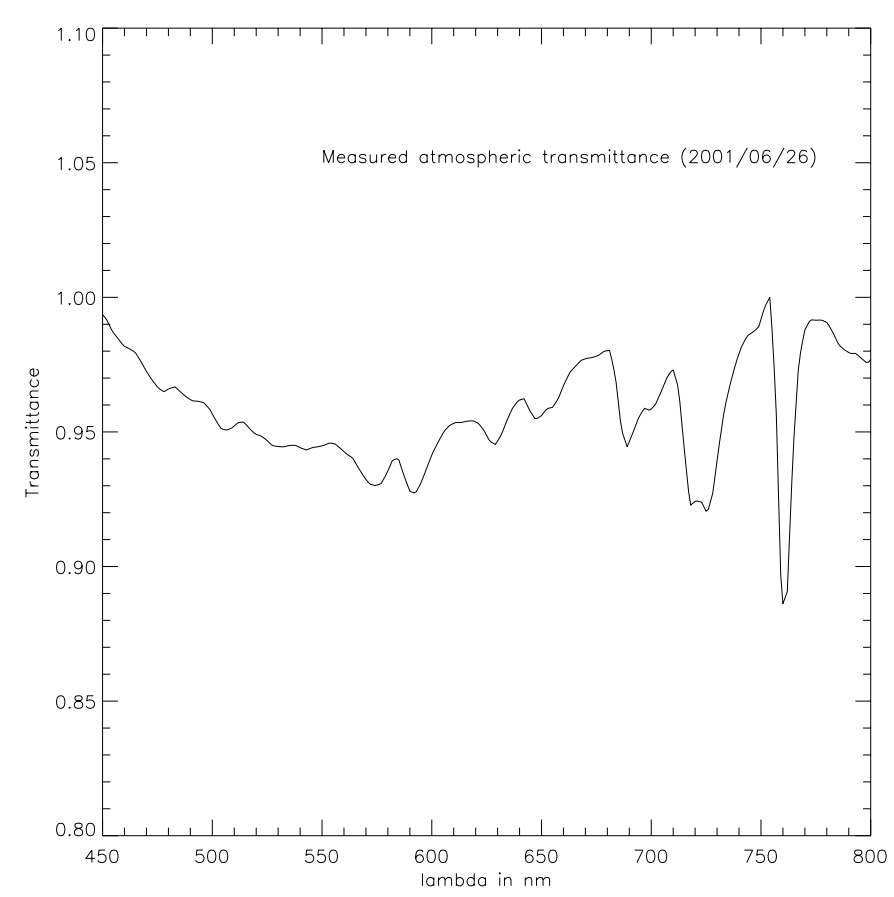

Fig. 3. An example of a measured 1-airmass atmospheric transmittance, after Rayleigh scattering correction. $\mathrm{H}_{2} \mathrm{O}$ and $\mathrm{O}_{2}$ bands are obviously present, while the broad ozone goes from 450 to $700 \mathrm{~nm}$ with maximum absorption at $\approx 600 \mathrm{~nm}$.

and then are transmitted back through the atmosphere a second time, giving a power of 2 on $A T(\lambda)$. Clearly $\alpha$ represents an airmass, but its value of 2 is a rough approximation: all photons do not cross twice an airmass of 1, depending on their impact location on Earth, on how they are scattered in the Earth's atmosphere versus their wavelength, and again how the Sun-Earth-Moon triplet is configured (described by a timedependent vector $\boldsymbol{g}_{\mathbf{3}}$ ). Moreover, photons can be reflected by high-altitude clouds having a high albedo, thereby crossing a thinner airmass before going back to space. The latter proportion of photons is also time-dependent, thus implying that $\alpha=\alpha\left(\boldsymbol{g}_{3}, \lambda, t\right)$ is probably difficult to estimate.

We obtained $A T(\lambda)$ by the ratio of two mean spectra $M S(\lambda)$ taken at two different Moon elevations. This obviously gives only a measure of the local atmosphere transmittance, whereas $A T(\lambda)$ in Eq. (4) represents a mean spectrum for the illuminated Earth seen from the Moon.

Our measured $A T(\lambda)$ is corrected for Rayleigh scattering and is normalized to 1 airmass (Fig. 3). Then Eq. (4) gives

$S R(\lambda)=\frac{E A(\lambda)}{A T^{\alpha}(\lambda)}$

We adjusted $\alpha$ to remove the atmospheric bands in $S R(\lambda)$ between 600 and $800 \mathrm{~nm}$. We found $\alpha$ values between 1 and 3 . Most of the time, we treated the $\mathrm{O}_{2}, \mathrm{O}_{3}$ and $\mathrm{H}_{2} \mathrm{O}$ bands separately with different $\alpha$ to obtain the best correction.

The surface reflectance we obtain in practice from Eq. (5) is shown in Fig. 4. The albedo spectrum of the ocean is $\approx 0.1$ at $500 \mathrm{~nm}$ and decreases smoothly to $\approx 0.05$ at $750 \mathrm{~nm}$ (McLinden et al. 1997, Fig. 4). But considering these values and the land albedo from Fig. 1, associated with a typical cloud 


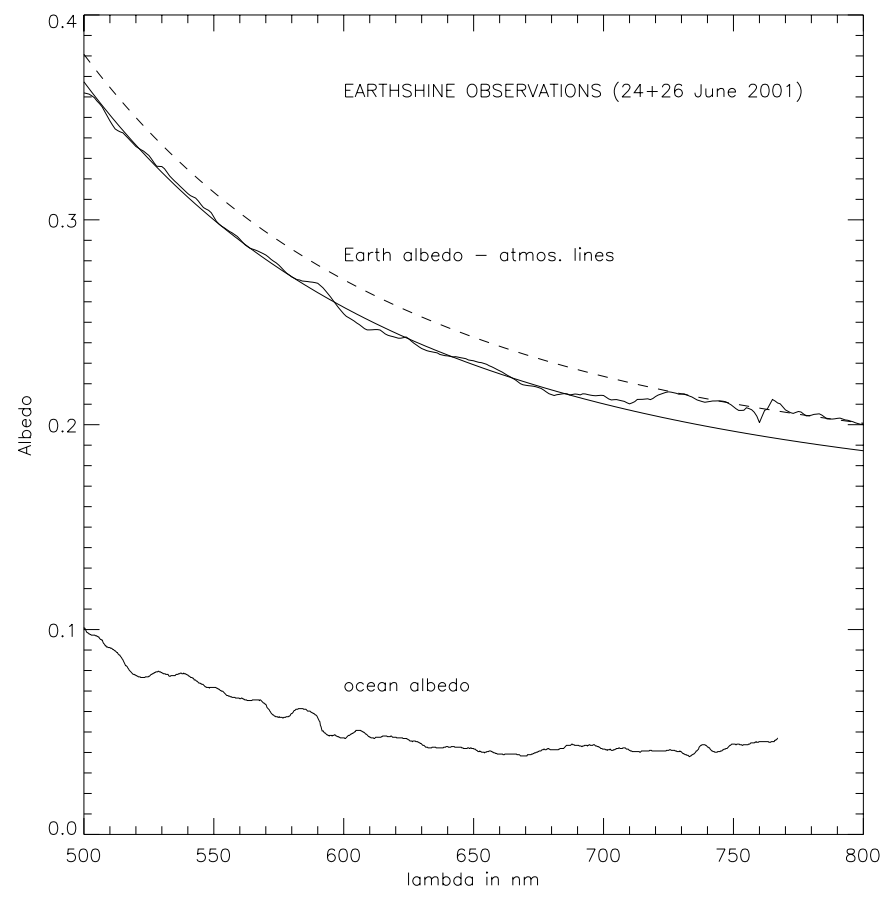

Fig. 4. An example of Rayleigh correction: the graph shows the $24+26$ June spectrum $S R(\lambda)$ (above) after atmospheric absorption correction (Eq. (5)), but still containing the Rayleigh scattering signature. The spectrum is fitted with a Rayleigh law adjusted over the $[500 ; 670 \mathrm{~nm}$ ] window. The fit is then translated (dash) and adjusted to the $[740 ; 800 \mathrm{~nm}$ ] region of $S R(\lambda)$ to show the $V R E$ (here $V R E=7 \%$ ). $S R(\lambda)$ is normalized to 0.3 at $550 \mathrm{~nm}$ (Goode et al. 2001) to compare to the ocean albedo (McLinden et al. 1997). The $S R(\lambda)$ higher slope in the blue is the signature of Rayleigh diffusion in Earth's atmosphere.

cover of $50 \%$ for both ocean and land, it can be shown that the higher Earth albedo in the blue in Fig. 2 cannot be explained by the higher albedo of the ocean in the blue, but rather by a contribution of Rayleigh diffusion in the atmosphere.

Therefore $S R(\lambda)$ does not represent the pure surface reflectance, but includes uncorrected atmospheric scattering (for simplicity, we nevertheless continue to name $S R(\lambda)$ the result of Eq. (5)). The Fig. 4 shows $S R(\lambda)$ fitted with the Rayleigh law $A+B / \lambda^{4}$ adjusted over the $[500 ; 670 \mathrm{~nm}]$ window. The slope towards the blue does not hide the relatively sharp vegetation signature, which appears around $700 \mathrm{~nm} . S R(\lambda)$ is then normalized to the Rayleigh fit (Fig. 5).

To quantify the vegetation signature, we define the Vegetation Red Edge (VRE) as

$V R E=\frac{r_{I}-r_{R}}{r_{R}}$

where $r_{R}$ and $r_{I}$ are the mean reflectances in the [600;670 nm] and $[740 ; 800 \mathrm{~nm}$ ] windows in the spectrum after it has been flattened with a Rayleigh law as explained above. This VRE definition giving the relative height of the step due to the vegetation is close to the NDVI (Normalized Difference Vegetation Index, Rouse et al. 1974; Tucker 1979) used in Earth satellite observation which considers the difference, after atmospheric

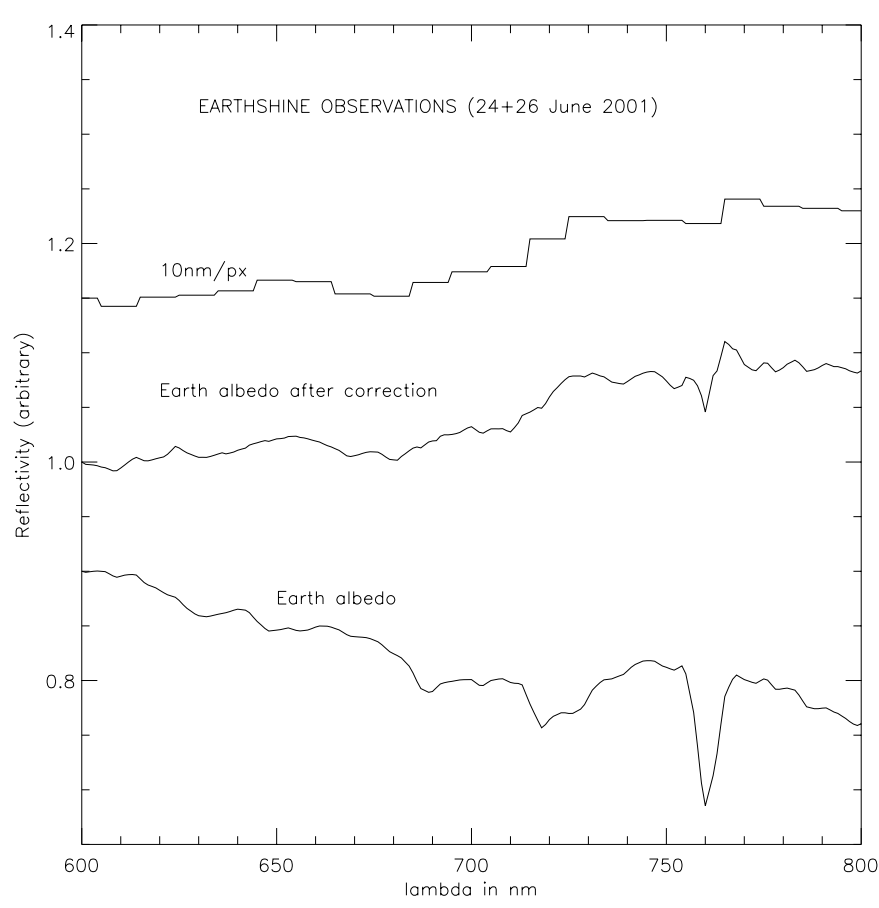

Fig. 5. An example of data reduction sequence: the graph shows the June albedo spectrum $E A(\lambda)$ (bottom). All atmospheric absorption features are then corrected according to Eq. (5) and the spectrum is flattened with a Rayleigh law adjusted in the $[500 ; 670 \mathrm{~nm}]$ window (Fig. 4). The result is shown above with 1 and $10 \mathrm{~nm} / \mathrm{px}$ resolution. The measured red edge around $700 \mathrm{~nm}$ is $V R E=7 \%$ (Eq. (6)).

correction, between the reflected fluxes in broad red and infrared bands, normalized to the sum of the fluxes in these bands,

$N D V I=\frac{f_{I}-f_{R}}{f_{R}+f_{I}}$

Flattened $S R(\lambda)$ spectra are shown in Figs. 6 and 7. Equation (6) gives $V R E$ values ranging between 4 and $10 \%$.

\section{Discussion}

First, we verified that the Moon albedo cancels correctly in practice when we do the ratio $E A(\lambda)=E S(\lambda) / M S(\lambda)$ (Eq. (3)). Since $M S(\lambda)$ is the mean of 10 long-slit Moonlight spectra totalling $20^{\prime}$ while a single Earthshine $\operatorname{ES}(\lambda)$ spectra is only $2^{\prime}$, we computed the ratio of 2 spectra $\left(2^{\prime} / 20^{\prime}\right)$ of Moonlight $M S(\lambda)$ taken on different Moon regions. We obtain a constant with $\sigma<0.5 \%$ meaning that the Moon albedo cancels correctly in Eq. (3).

We also verified that the second order spectrum pollution is negligible: it has been measured to be $0 \%$ at $760 \mathrm{~nm},<0.3 \%$ at $780 \mathrm{~nm}$ and $<0.5 \%$ at $800 \mathrm{~nm}$.

To test our VRE measurements, we also measured the $V R E$ on spectra of Vega and the sunlit Moon, for which we obviously should have $V R E=0 \%$. After standard bias, dark and flat corrections, the data are calibrated with reference A0V and Sun spectra, respectively, then flattened by normalization to a black body curve. Vega spectra show a $V R E=-1 \%$, $\sigma=2 \%$. We also used the standard Moon albedo spectrum 


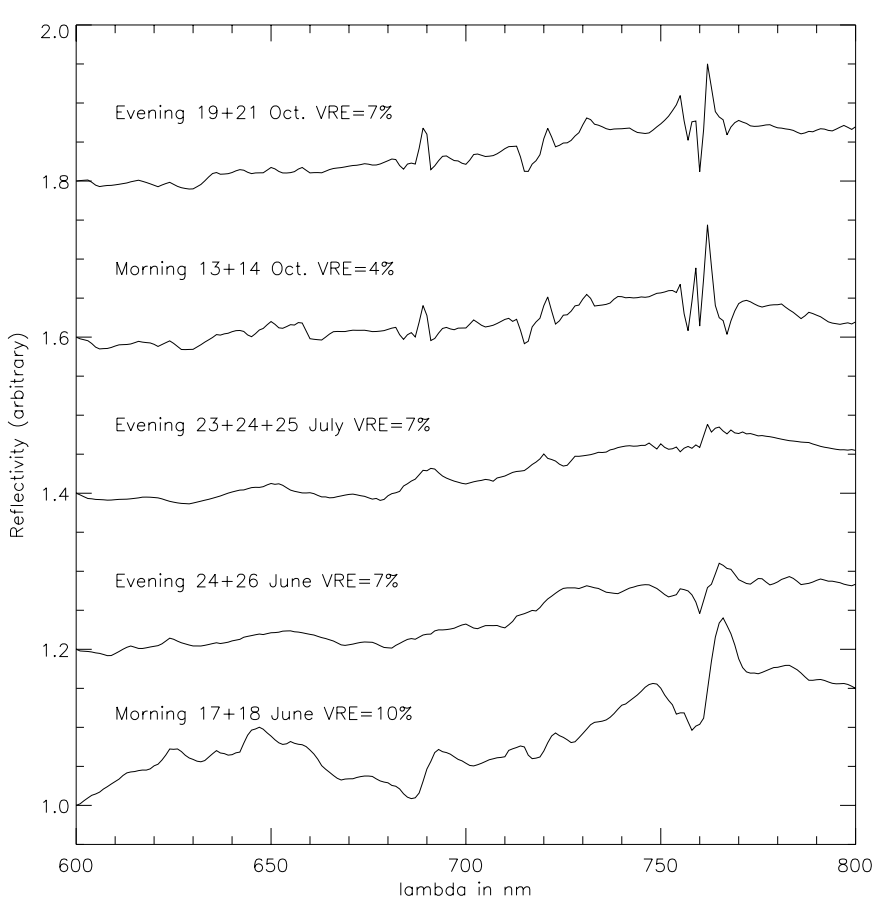

Fig. 6. Collection of $S R(\lambda)$ spectra normalized to 1 at $600 \mathrm{~nm}$, but shifted upwards for clarity by $0.2,0.4,0.6$ and 0.8 , respectively. Note that only the $[600 ; 670]$ and $[740 ; 800 \mathrm{~nm}]$ windows are used to estimate the $V R E$.

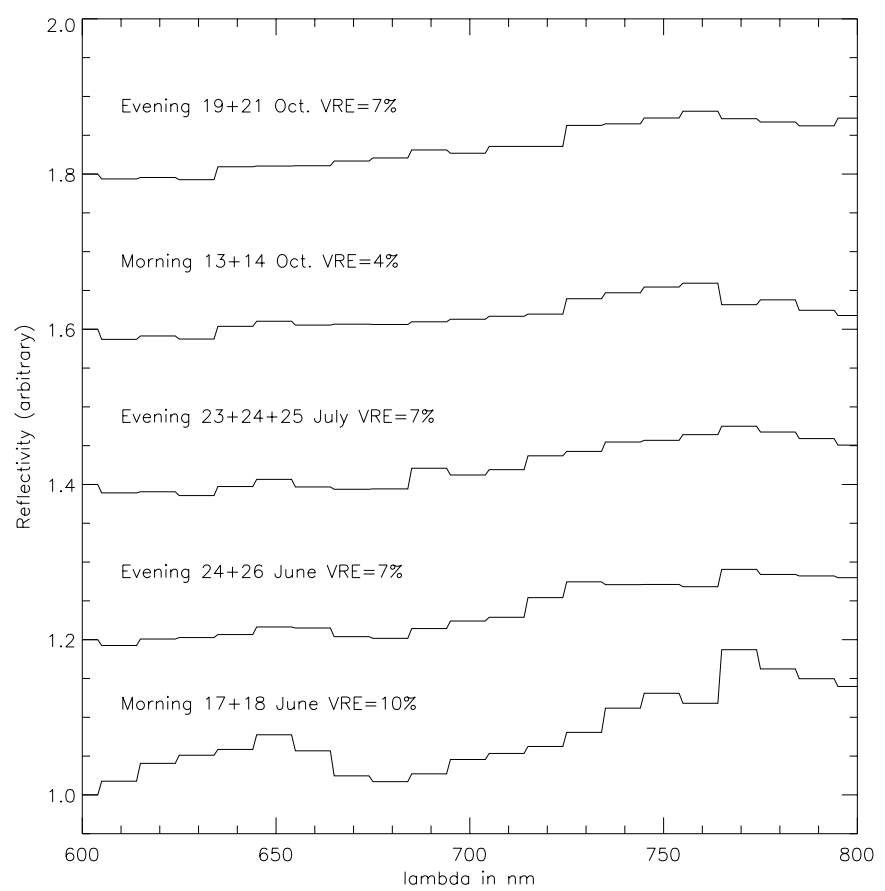

Fig. 7. The same collection of $S R(\lambda)$ spectra as in Fig. 6 but binned to $10 \mathrm{~nm} / \mathrm{px}$.

of Apollo16 sample 62231 (Pieters 1999) to properly flatten the Moon data: we obtain $V R E=0 \%, \sigma=3 \%$. Therefore, we conclude that $i$ ) the $V R E$ on these sources is measured to be $V R E=0 \%$, with $\sigma \approx 3 \%$, and $i i)$ the $V R E$ we measured in the Earth albedo ranges between 4 and $10 \%$, with a similar error of $\sigma \approx 3 \%$ (Table 3). In the June $17+18$ spectrum, we probably
Table 3. Results for the measured Vegetation Red Edge VRE.

\begin{tabular}{lll}
\hline \hline Epoch & Viewed zone & VRE \\
\hline $2001 / 06 / 17$ and 18 morning & Eur./Asia & $10 \pm 5 \%$ \\
$2001 / 06 / 24$ and 26 evening & Amer./Pacific & $7 \pm 3 \%$ \\
$2001 / 07 / 23,24$ and 25 evening & Amer./Pacific & $7 \pm 3 \%$ \\
$2001 / 10 / 13$ and 14 morning & Eur./Asia & $4 \pm 3 \%$ \\
2001/10/19 and 21 evening & Amer./Pacific & $7 \pm 3 \%$ \\
\hline
\end{tabular}

have a larger error on the $V R E$ due to the lack of bracketing from Earthshine with Moonlight spectra (Figs. 6 and 7).

These results are in agreement with estimations from models: Des Marais et al. (2001) predict a vegetation signature of "2\%, maybe larger if a large forested area is in view". Preliminary estimates gave 5\% (Schneider 2000a; Schneider 2000b), while our model described hereafter predicts $\approx 10 \%$.

Only green lands observed by different Earth observing satellites show this spectral feature around $700 \mathrm{~nm}$. It is thus legitimate to attribute our global $V R E$ to the terrestrial vegetation. Let us investigate this hypothesis a little further.

Table 4 shows which portion of the Earth is seen from the Moon at the time the observations were done. An important contribution to the Earth albedo comes from clouds. When clouds cover land without vegetation or an ocean area, its albedo adds to the general planet albedo, without suppressing the vegetation contribution, and simply reduces the global VRE. When clouds cover a forest region, the vegetation contribution to the $V R E$ for that Earth region is removed. The global VRE thus obviously depends not only on the global cloud cover, but also on which regions are covered by clouds during the observation. We have therefore taken cloud cover images available from http://wwwghcc.msfc.nasa.gov/GOES/globalir.html for each of our observation dates and have estimated for each run of observation the fraction of land and ocean covered by clouds (Table 5).

One can a priori estimate the VRE from the albedo and relative surfaces of ocean, land and cloud. Using Eq. (6), the $V R E$ can be written

$$
V R E_{\text {th }}=\frac{A_{1}\left(r_{I}-r_{R}\right)_{1} C F_{1}}{A_{\mathrm{o}}\left(r_{R}\right)_{\mathrm{o}} C F_{\mathrm{o}}+A_{1}\left(r_{R}\right)_{1} C F_{1}+C C\left(r_{R}\right)_{\mathrm{c}}}
$$

where $C F_{\mathrm{o}, 1}$ are the fractional cloud-free areas for ocean and land (from deserts to green forests), respectively, and $A_{\mathrm{o}, 1}$ are the corresponding areas in view from the Moon. $C C$ is the fractional cloud cover, equal to $C C=\left(1-C F_{\mathrm{o}}\right) A_{\mathrm{o}}+\left(1-C F_{1}\right) A_{1}$. Calculating a mean $V R E_{\text {th }}$ for Earth with $A_{0}=0.7$ and $A_{1}=0.3$ (standard Earth ocean/land ratio), $\left(r_{\mathrm{I}}-r_{R}\right)_{1}=0.15$ (based on $N D V I$ data), $\left(r_{R}\right)_{\mathrm{o}}=0.05,\left(r_{R}\right)_{1}=0.25$ and $\left(r_{R}\right)_{\mathrm{c}}=0.5$ (albedos for ocean, land and cloud), we find, for a mean cloud cover of $50 \%$ for all regions, $V R E_{\mathrm{th}} \approx 7 \%$. We estimate the error on $V R E_{\text {th }}$ to be $\pm 3 \%$ (cloud cover estimation, seasonal variation of the difference $\left(r_{\mathrm{I}}-r_{R}\right)_{1}$, vegetation mutual shadow effects). The small contribution of ocean chlorophyll is also neglected here. 
Table 4. Earth zone viewed from the Moon at the epochs of observations.

\begin{tabular}{llll}
\hline \hline Epoch & Viewed zone & \multicolumn{2}{l}{ Land Ocean } \\
$\%$ & $\%$ \\
\hline $2001 / 06 / 17$ and 18 & Eur./Asia & 50 & 50 \\
$2001 / 06 / 24$ and 26 & Amer./Pacific & 40 & 60 \\
$2001 / 07 / 23,24$ and 25 & Amer./Pacific & 40 & 60 \\
$2001 / 10 / 13$ and 14 & Eur./Asia & 50 & 50 \\
$2001 / 10 / 19$ and 21 & Amer./Pacific & 30 & 70 \\
\hline
\end{tabular}

The resulting $V R E_{\text {th }}$ computed from the Earth phase seen from the Moon (Table 4) and cloud cover (Table 5) are given in Table 6. The denominator of Eq. (8) represents an albedo, known to be 0.30 for the Earth (Goode et al. 2001), and its value here ranges between 0.30 and 0.34 depending on the date of observation. The $V R E_{\text {th }}$ values are in acceptable agreement with preliminary estimates of $5 \%$ (Schneider 2000a; Schneider 2000b), but are higher than our observations. This partially comes from terrestrial limb and directionality of the vegetation reflectance effects. The latter are complicated (Privette et al. 1995; Hapke et al. 1996), due for instance to mutual shadow effects between trees.

\section{Conclusion}

Although it seems that the Earth's vegetation signature might be visible as a red edge at $700 \mathrm{~nm}$, it is difficult to measure in the Earthshine for two reasons. The first reason is related to its variable amplitude, induced by a variable cloud cover and Earth phase. The second reason is because it is hidden below strong atmospheric bands which need to be removed to access the surface reflectance including the vegetation signature.

For the Earth, our knowledge of different surface reflectivities (deserts, ocean, ice etc.) help us to assign the $V R E$ of the Earthshine spectrum to terrestrial vegetation. For an exoplanet, a $V R E$-like index might be as difficult to measure as for the Earth due to variable cloud cover of the planet. Even if an extrasolar planet would give a clear $V R E$-like spectral signal, its use as a biosignature would raise some questions because:

1/ For several organisms (such as Rhodopseudonomas, Blankenship et al. 1995) the "red edge" is not at $700 \mathrm{~nm}$, but at $1100 \mathrm{~nm}$.

2/ Some rocks, like schists, may have a similar spectral feature. For instance, spectra of Mars show a similar spectral feature at $3.5 \mu \mathrm{m}$, which were erroneously interpreted as vegetation due to their similarity with lichen spectra (Sinton 1957).

We nevertheless believe that, associated with the presence of water (and secondarily oxygen) and correlated with seasonal variations, a vegetation-like spectral feature would provide more insight than simply oxygen on the bio-processes possibly taking place on the planet. But since water, and thus clouds and rain, are essential for the growth of vegetation, extrasolar planets with a very low cloud cover and a corresponding high vegetation index are unlikely, more especially if the planet is seen pole-on, with a bright white polar cover.
Table 5. Cloud cover estimated for the Earth zone viewed from the Moon at the epochs of observations.

\begin{tabular}{lcc}
\hline \hline Epoch & $\begin{array}{c}\text { Land } \\
\left(1-C F_{1}\right) \%\end{array}$ & $\begin{array}{c}\text { Ocean } \\
\left(1-C F_{\mathrm{o}}\right) \%\end{array}$ \\
\hline 2001/06/17 and 18 morning & 60 & 50 \\
2001/06/24 and 26 evening & 40 & 50 \\
2001/07/23, 24 and 25 evening & 50 & 50 \\
2001/10/13 and 14 morning & 50 & 50 \\
2001/10/19 and 21 evening & 50 & 50 \\
\hline
\end{tabular}

Table 6. Comparison of the observed and calculated Vegetation Red Edge VRE.

\begin{tabular}{lccc}
\hline \hline Epoch & Viewed zone & $V R E_{\mathrm{obs}}$ & $V R E_{\mathrm{th}}$ \\
\hline $2001 / 06 / 17$ and 18 morning & Eur./Asia & $10 \pm 5 \%$ & $9 \pm 3 \%$ \\
$2001 / 06 / 24$ and 26 evening & Amer./Pacific & $7 \pm 3 \%$ & $12 \pm 3 \%$ \\
$2001 / 07 / 23,24$ and 25 evening & Amer./Pacific & $7 \pm 3 \%$ & $10 \pm 3 \%$ \\
$2001 / 10 / 13$ and 14 morning & Eur./Asia & $4 \pm 3 \%$ & $12 \pm 3 \%$ \\
2001/10/19 and 21 evening & Amer./Pacific & $7 \pm 3 \%$ & $7 \pm 3 \%$ \\
\hline
\end{tabular}

On the other hand, an extrasolar planet vegetation surface could be larger than on Earth (like during periods in the paleozoic and mesozoic eras on Earth for example).

One must also note that the measurement of an extrasolar planet $V R E$ will not suffer from the intrinsic difficulty of the same measurement for the Earth through the Earthshine spectrum: the extrasolar planet albedo will simply be given by the ratio of spectra planet/mother star. But a model of the exoplanet atmosphere is necessary to be able to remove the absorption bands that may partially hide the vegetation. Although the probability is weak, the planet may occult a background star, thus providing us a direct measurement of the planet atmosphere absorption.

The detection of a VRE index between 0 and $10 \%$ requires a photometric precision better than $3 \%$. Exposure time to achieve this precision with Darwin/TPF on an Earth-like planet at $10 \mathrm{pc}$ with a spectral resolution of 25 is of the order of $100 \mathrm{~h}$ based on recent simulations (Riaud et al. 2002).

Finally, the Earth albedo spectral variations study is of interest for global Earth observation. It might provide data on climate change, as broad-band measurements recently showed (Goode et al. 2001). We also think that the spectrum of Earthshine might be used for example to monitor the global ozone (with the Chappuis or Huggins bands).

During the submission of the paper, we have been informed of a similar work by Woolf et al. (2002).

Acknowledgements. We are grateful to P. François who took test spectra of the Earthshine in 1999. We thank Ph. Gastellu-Etchegorry for providing us Earth observation satellite data. We also thank C. Prigent, L. Beaufort, G. Bellucci, D. Gillet, S. Le Mouélic, A. Labeyrie, J. M. Perrin and referee S. Franck for constructive remarks about this work. F. Valbousquet from Optique et Vision lent us some parts for the spectrograph. 


\section{References}

Angel, J. R. P., Chen, A. Y. S., \& Woolf, N. J. 1986, Nature, 322, 341 Angel, J. R. P., \& Woolf, N. J. 1997, ApJ, 475, 373

Arcichovsky, V. M. 1912, Auf der Suche nach Chlorophyll auf den Planeten in Annales de l'Institut Polytechnique Don Cesarevitch Alexis a Novotcherkassk, 1(17), 195

Beichman, C., Lindensmith, C., \& Woolf, N. J. 1999, The Terrestrial Planet Finder (JPL Publication), 99-3

Blankenship, R. E., Madigan, M. T., \& Bauer, C. E. 1995, Anoxygenic Photosynthetic Bacteria (Kluwer Academic Publishing, Dordrecht, The Netherlands)

BRDF (Special issue on) 2000, Remote Sensing Rev., 18, 83 to 551

Clark, R. N. 1999, Manual of Remote Sensing, ed. A. Rencz (J. Wiley and Sons, New-York)

Coliolo, F., Labeyrie, A., \& Schneider, J. 2000, in Proc., Sixth Trieste Conference on Chemical Evolution First Steps in the Origin of Life in the Universe

Danjon, A. 1928, Ann. Obs. Strasbourg, 2, 165

Des Marais, D., Harwit, M., Jucks, K., et al. 2001, JPL Publication, 01-008

Goode, P., Qiu, J., Yurchyshyn, V., et al. 2001, Geophys. Res. Lett., 28(9), 1671

Hapke, B., Dimucci, D., Nelson, R., \& Smythe, W. 1996, Lunar Planet. Sci., 27, 491

Kelley, K. W., \& the Association of Space Explorers 1988, The Home Planet (Addison-Wesley Publishing Company, New-York)

Labeyrie, A., Schneider, J., Boccaletti, A., et al. 1999, in ESA Proc., Darwin and Astronomy, ESA SP-451, 21

Labeyrie, A. 1999, in Proc., Planets Outside the Solar System: Theory and Observations, ed. J. M. Mariotti, \& D. Alloin, NATO ASI, 532,261

Léger, A., Mariotti, J. M., Menesson, B., et al. 1996, Icarus, 123, 249
Liang, S., \& Strahler, A. H. 1999, The Earth Obs., 11, 27

Lovelock, J. 1975, Proc. Roy. Soc. London, B 189, 167

McLinden, C. A., McConnell, J. C., Griffioen, E., McElroy, C. T., \& Pfister, L. 1997, J. Geophys. Res., 102, 18801

Owen, T. 1980, in Proc., Strategies for the search for life in the universe, ed. M. Papagiannis (Reidel), 177

Pieters, C. M. 1999, in Proc., New Views of the Moon II: Understanding the Moon Through the Integration of Diverse Datasets, September 22-24, 1999, Flagstaff, Arizona, abstract 8025 (http://www.planetary.brown.edu/pds/ AP62231.html)

Privette, J. L., Myneni, R. B., Emery, W. J., \& Pinty, B. 1995, J. Geophys. Res., 100, 25497

Rouse, J. W., Haas, R. H., Schell, J. A., \& Deering, D. W. 1974, Final Report, type III, NASA/GSFC, Greenbelt

Riaud, P., Boccaletti, A., Gillet, S., et al. 2002, A\&A, accepted

Sagan, C., Thompson, W. R., Carlson, R., Gurnett, D., \& Hord, C. 1993, Nature, 365, 715

Schneider, J. 2000a, Exoplanets in A Encyclopaedia of Astronomy and Astrophysics (Institute of Physics Publishing)

Schneider, J. 2000b, private communication

Schneider, J., Auvergne, M., Baglin, A., et al. 1998, in ASP Conf. Ser., 148, ed. Charles E. Woodward, J. Michael Shull, \& Harley A. Thronson, Jr., 298

Sinton, W. 1957, ApJ, 126, 231S

Tikhoff, G. A. 1914, Mitteilungen der Nikolai-Hauptsternwarte zu Pulkowo, No. 62, Band $\mathrm{VI}_{2}, 15$

Tucker, C. J. 1979, Remote Sensing of the Environment, 8, 127

Udry, S., \& Mayor, M. 2001, in ESA Proc., First European Workshop on Exo/Astrobiology, in press

Woolf, N., Smith, P., Traub, W., \& Jucks, K. 2002, ApJ, accepted

Wolszczan, A., \& Frail, D. 1992, Nature, 255, 145 